\title{
ANODIC SYNTHESIS, SPECTRAL CHARACTERIZATION AND ANTIMICROBIAL ACTIVITY OF NOVEL 2-AMINO-5-SUBSTITUTED-1,3,4-OXADIAZOLES
}

\author{
SANJEEV KUMAR* \\ Department of Chemistry, Iswar Saran Degree College (University of Allahabad) \\ Allahabad-211004, India
}

(Received: July 29, 2009 - Accepted: October 21, 2009)

\begin{abstract}
Synthesis of 2-amino-5-substituted-1,3,4-oxadiazoles through the electrochemical oxidation of semicarbazone was carried out at platinum anode at room temperature under controlled potential electrolysis in an undivided cell assembly. The electrolysis were carried out in the non aqueous medium acetic acid and lithium perchlorate was used as a supporting electrolyte. The antimicrobial activities of the compounds have been studied by screening the compounds against two gram negative organisms Klebsiella pneumoniae, Escherichia coli, and two gram positive organisms Bacillus subtilis and Streptococcus aureus and antifungal activity against Aspergillus niger and Crysosporium pannical and results have been compared with the standard antibacterial Streptomycin and antifungal Griseofulvin.
\end{abstract}

Keywords: Semicarbazone, Oxadiazoles, Electrolysis, Antimicrobial studies

\section{INTRODUCTION}

1,3,4-oxadiazole is a versatile lead molecule for designing potential bioactive agents. The 1,3,4-oxadiazole derivatives have been found to exhibit diverse biological activities such as hypotensive ${ }^{1}$ anti-microbial ${ }^{2-4}$, anti-HIV ${ }^{2-4}$, anti-fungal ${ }^{5-6}$ anti-inflammatory ${ }^{7}$ antimitotic activity ${ }^{8}$ and muscle relaxant ${ }^{9}$

It have been found in the literature study that the methods for synthesis of oxadiazole 1 include bromine oxidation of semicarbazide derivative and the cyclodesulfurization of acylthiosemicarbazide derivatives in the solution using $\mathrm{I}_{2} / \mathrm{NaOH}$ or 1,3-dicyclohexylcarbodimide (DCC) $)^{10-13}$ as well as mercury(II) acetate $\left(\mathrm{Hg}(\mathrm{OAc})_{2}\right)$ or yellow mercury(II) oxide $\mathrm{HgO}^{14-16}$ Evans $^{17}$ synthesized oxadiazole derivatives by rapid parallel synthesis in efficient onepot preparation using resin-bound reagents. All these methods are usually carried out in various different synthetic steps and require the heating at higher temperature. The handling of these reagents is not only difficult but also very hazardous to environment. The each stage of the reaction including extraction and purification of the products from the mixture requires great precautions.

These reports including our earlier work ${ }^{18-23}$ prompted us to synthesize 2-amino-5-substituted-1,3,4-oxadiazole derivatives through the electrochemical oxidation of semicarbazone 4 in the hope of getting potent biodynamic agents and evaluate their antimicrobial activity.

\section{EXPERIMENTAL}

\section{Analysis and measurements}

The reagents used for the preparation of oxadiazoles were of Merck products. Spectroscopic grade solvents were used for spectral measurements. The carbon, hydrogen and nitrogen contents in each sample were performed at RSIC, CDRI Lucknow. The IR spectra were recorded on a Perkin-Elmer 783 spectrophotometer $\mathrm{KBr}$ pellets. ${ }^{1} \mathrm{H}$ NMR and ${ }^{13} \mathrm{C}$ NMR spectra were recorded on a Bruker DRX $300(300 \mathrm{MHz}) \mathrm{FT}$ spectrometer in $\mathrm{CDCl}_{3}$ using TMS as internal reference. Chemical shifts are reported in ppm downfield from TMS as internal reference. Elemental analyses were carried out in the Elementar VarioEL III instrument. Melting points were determined on a VEB Wagetechink Rapio PHMK05 instrument and are uncorrected. Water used was doubly distilled.

General procedure for the synthesis of compounds (1a-1n)

Semicarbazide hydrochloride $3(9.0 \mathrm{mmol})$ and sodium acetate $(12.2$ mmol) was dissolved in water $(10 \mathrm{~mL})$ and then aldehyde $2(4.16 \mathrm{mmol})$ was added with continuous stirring. The mixture was left overnight, which gives semicarbazone 4. Now semicarbazone $\mathbf{4}(12.25 \mathrm{mmol})$, lithium perchlorate $(1.3$ $\mathrm{mmol})$ and acetic acid $(200 \mathrm{~mL})$ was taken in the reaction cell assembly with platinum plate as working as well as counter electrode and saturated calomel electrode as reference electrode. Controlled potential electrolysis ${ }^{24-31}$ were performed at their corresponding oxidation potentials at room temperature, tabulated as Table 1. The product was extracted from the acetic acid solution to chloroform by the simple solvent extraction technique. 4-6 $\mathrm{Fmol}^{-1}$ of electricity was passed for the electrolysis, which is very small in comparison to energy used in other conventional methods.
Table 1. Anodic synthesis of 2-amino-5-substituted-1,3,4-oxadiazole derivatives

\begin{tabular}{|c|c|c|c|c|c|c|}
\hline Entry & $\mathrm{R}$ & $\begin{array}{l}\text { Time } \\
{[\mathrm{h}]}\end{array}$ & Product & $\begin{array}{c}\text { Applied } \\
\text { Potential } \\
\text { [V] }\end{array}$ & $\begin{array}{l}\text { Current } \\
{[\mathrm{A}]}\end{array}$ & $\begin{array}{c}\text { Yield } \\
{[\%]}\end{array}$ \\
\hline 1 & $3-\mathrm{MeC}_{6} \mathrm{H}_{4}$ & 3 & $1 \mathrm{a}$ & 1.60 & 0.15 & 90 \\
\hline 2 & $3-\mathrm{C}_{6} \mathrm{H}_{5} \mathrm{COC}_{6} \mathrm{H}_{4}$ & 4 & $1 \mathrm{~b}$ & 1.90 & 0.18 & 85 \\
\hline 3 & $5-\mathrm{Br}, 2-\mathrm{OHC}_{6} \mathrm{H}_{3}$ & 4 & $1 \mathrm{c}$ & 2.00 & 0.17 & 75 \\
\hline 4 & $\begin{array}{c}3-\left(4-\mathrm{Me}_{3} \mathrm{CC}_{6} \mathrm{H}_{4} \mathrm{O}\right) \\
\mathrm{C}_{6} \mathrm{H}_{4}\end{array}$ & 3 & $1 d$ & 1.75 & 0.19 & 92 \\
\hline 5 & $4-(\mathrm{COOH}) \mathrm{C}_{6} \mathrm{H}_{4}$ & 5 & $1 \mathrm{e}$ & 2.10 & 0.20 & 70 \\
\hline 6 & 10-Cl Anthryl & 4 & $1 \mathrm{f}$ & 1.95 & 0.15 & 88 \\
\hline 7 & $5-\mathrm{Cl}, 2-\left(\mathrm{NO}_{2}\right) \mathrm{C}_{6} \mathrm{H}_{3}$ & 5 & $1 \mathrm{~g}$ & 2.20 & 0.17 & 80 \\
\hline 8 & $5-\mathrm{Cl}, 2-\mathrm{OHC}_{6} \mathrm{H}_{3}$ & 5 & $1 \mathrm{~h}$ & 1.80 & 0.18 & 83 \\
\hline 9 & $4-(\mathrm{CN}) \mathrm{C}_{6} \mathrm{H}_{4}$ & 4 & $1 \mathrm{i}$ & 1.80 & 0.19 & 78 \\
\hline 10 & $3,5-\mathrm{Br}_{2} \mathrm{C}_{6} \mathrm{H}_{3}$ & 4 & $1 \mathrm{j}$ & 1.65 & 0.18 & 81 \\
\hline 11 & $\begin{array}{c}\text { 3,5-( }\left(\mathrm{Me}_{3} \mathrm{C}\right)_{2} \\
4-\mathrm{OHC}_{6} \mathrm{H}_{2}\end{array}$ & 3 & $1 \mathrm{k}$ & 1.60 & 0.11 & 86 \\
\hline 12 & $2,4-\mathrm{Cl}_{2} \mathrm{C}_{6} \mathrm{H}_{3}$ & 5 & 11 & 1.95 & 0.18 & 82 \\
\hline 13 & $2,3-\mathrm{F}_{2} \mathrm{C}_{6} \mathrm{H}_{3}$ & 5 & $1 \mathrm{~m}$ & 2.10 & 0.21 & 79 \\
\hline 14 & 2,6- $-\mathrm{F}_{2} \mathrm{C}_{6} \mathrm{H}_{3}$ & 5 & $1 \mathrm{n}$ & 2.20 & 0.20 & 78 \\
\hline
\end{tabular}

The spectral data of compounds, $\mathbf{1 a - 1} \mathbf{n}$ is given below.

2-amino-5-methylphenyl-1,3,4-oxadiazole (1a) Light brown needles, $\mathrm{m}$. p. $74-75{ }^{\circ} \mathrm{C}$; IR cm ${ }^{-1} 3444,3045,3010,2965,2927,1602(\mathrm{C}=\mathrm{N}-\mathrm{N}=\mathrm{C}), 1265$, 1069 (C-O-C), 960, 765; H $\mathrm{H}^{1} \mathrm{NMR}\left(400 \mathrm{MHz}, \mathrm{CDCl}_{3}\right) 7.75$ (s, 2H, NH ), 6.52$7.24(\mathrm{~m}, \mathrm{~J}=2.6$ and $5.6 \mathrm{~Hz}, 4 \mathrm{H}), 1.12\left(\mathrm{~s}, 3 \mathrm{H}, \mathrm{CH}_{3}\right) ; \mathrm{C}^{13} \mathrm{NMR}\left(75 \mathrm{MHz}, \mathrm{CDCl}_{3}\right)$ 
178.2, 149.9, 138.6, 141.3, 136.5, 129, 127.8, 126.5, 20.6; m/z (ES-MS) 173.0, Mol. Formula $\mathrm{C}_{9} \mathrm{H}_{7} \mathrm{~N}_{3} \mathrm{O}$, Mol. Wt.: 173.2, Calculated C, 62.42; H, 4.04; N, 24.27, found $\mathrm{C}, 62.12 ; \mathrm{H}, 4.00 ; \mathrm{N}, 23.86$

2-amino-5-(3-benzoyloxy)phenyl-1,3,4-oxadiazole (1b) Pale yellow needles, m. p. $72-74{ }^{\circ} \mathrm{C}$; IR cm ${ }^{-1} 3350,3045,1755,1617(\mathrm{C}=\mathrm{N}-\mathrm{N}=\mathrm{C}), 1269$, 1065 (C-O-C), 910, 860; $\mathrm{H}^{1} \mathrm{NMR}\left(400 \mathrm{MHz}, \mathrm{CDCl}_{3}\right) 7.75$ (s, 2H, NH ), 7.25$7.69(\mathrm{~m}, \mathrm{~J}=2.6$ and $5.6 \mathrm{~Hz}, 9 \mathrm{H})$; $\mathrm{C}^{13} \mathrm{NMR}\left(75 \mathrm{MHz}, \mathrm{CDCl}_{3}\right) 172,149.5,137.1$, $136.3,132.9,131.3,130.2,128.7,128.1,128.0,127.8 ; \mathrm{m} / \mathrm{z}$ (ES-MS) 264.8, Mol. Formula $\mathrm{C}_{15} \mathrm{H}_{11} \mathrm{~N}_{3} \mathrm{O}_{2}$, Mol. Wt.: 265.3, Calculated C, 67.92; H, 4.14; N, 15.84, found $\mathrm{C}, 67.45 ; \mathrm{H}, 4.03 ; \mathrm{N}, 15.44$.

2-amino-5-(5-bromo-4-hydroxy)phenyl-1,3,4-oxadiazole (1c) Black needles, m. p. $85-87^{\circ} \mathrm{C}$; IR cm ${ }^{-1} 3440,3360,3045,1604(\mathrm{C}=\mathrm{N}-\mathrm{N}=\mathrm{C}), 1267$, 1070 (C-O-C), 980, 890, 755, 655; $\mathrm{H}^{1} \mathrm{NMR}\left(400 \mathrm{MHz}, \mathrm{CDCl}_{3}\right) 7.75$ (s, 2H, $\left.\mathrm{NH}_{2}\right), 7.25-7.69(\mathrm{~m}, \mathrm{~J}=2.6$ and $5.6 \mathrm{~Hz}, 4 \mathrm{H}), 6.09(\mathrm{~s}, 1 \mathrm{H}, \mathrm{OH}) ; \mathrm{C}^{13} \mathrm{NMR}(75$ $\mathrm{MHz}, \mathrm{CDCl}_{2}$ ) 158.9, 147.2, 141.2, 136.4, 134.2, 129.7, 123.9, 123.7; m/z (ESMS) 265.2, Mol. Formula $\mathrm{C}_{10} \mathrm{H}_{8} \mathrm{~N}_{3} \mathrm{OBr}$, Mol. Wt.: 266.1, Calculated C, 45.11; $\mathrm{H}, 3.02 ; \mathrm{N}, 15.79 ; \mathrm{Br}, 30.07$, found C, 44.85; H, 2.75; N, 15.39; Br, 29.65 .

2-amino-5-(3-(4-tert-butyl phenoxy)phenyl-1,3,4-oxadiazole (1d) Yellow needles, m. p. 85-87 ${ }^{\circ} \mathrm{C}$; IR cm $\mathrm{cm}^{-1} 3444,3360,2860,1608(\mathrm{C}=\mathrm{N}-$ $\mathrm{N}=\mathrm{C}), 1270,1265,1072$ (C-O-C), 1130, 749.5; $\mathrm{H}^{1} \mathrm{NMR}\left(400 \mathrm{MHz}, \mathrm{CDCl}_{3}\right)$ $7.75\left(\mathrm{~s}, 2 \mathrm{H}, \mathrm{NH}_{2}\right), 6.52-7.14(\mathrm{~m}, \mathrm{~J}=2.6$ and $5.6 \mathrm{~Hz}, 8 \mathrm{H}), 1.3\left(\mathrm{~s}, 9 \mathrm{H}, \mathrm{CH}_{3}\right) ; \mathrm{C}^{13}$ NMR (75 MHz, $\left.\mathrm{CDCl}_{3}\right)$ 172.4, 167, 147.5, 140.2, 138.9, 137.5, 132.6, 128.5, 128.2, 128.1, 128.4, 122.8, 118.6, 114.6, 42.1, 20.6; m/z (ES-MS) 308.1, Mol. Formula $\mathrm{C}_{18} \mathrm{H}_{10} \mathrm{~N}_{3} \mathrm{O}_{2}$, Mol. Wt.: 308.5, Calculated C, 69.90; H, 6.14; N, 13.59, found $\mathrm{C}, 69.12 ; \mathrm{H}, 5.88 ; \mathrm{N}, 13.36$.

2-amino-5-(4-carboxy)phenyl-1,3,4-oxadiazole (1e) Brown needles, $\mathrm{m}$. p. 87-89 ${ }^{\circ} \mathrm{C}$; IR $\mathrm{cm}^{-1} 3360,3300,3060,1715,1612(\mathrm{C}=\mathrm{N}-\mathrm{N}=\mathrm{C}), 1280,1275$, 1065 (C-O-C), 962; $\mathrm{H}^{1} \mathrm{NMR}\left(400 \mathrm{MHz}^{\mathrm{CDCl}}{ }_{3}\right) 7.75$ (s, 2H, NH ), 6.52-7.24 $(\mathrm{m}, \mathrm{J}=2.6$ and $5.6 \mathrm{~Hz}, 4 \mathrm{H}) ; \mathrm{C}^{13} \mathrm{NMR}\left(75 \mathrm{MHz}, \mathrm{CDCl}_{3}\right) 172.6,144.9,141.5$, 141.2, 129.9, 129.5, 129.3; m/z (ES-MS) 204.6, Mol. Formula $\mathrm{C}_{9} \mathrm{H}_{7} \mathrm{~N}_{3} \mathrm{O}_{3}$, Mol. Wt.: 205.2, Calculated C, $52.68 ; \mathrm{H}, 3.41 ; \mathrm{N}, 20.48$, found C, $51.82 ; \mathrm{H}, 3.22$; N, 20.00 .

2-amino-5-(10-chloro)anthryl-1,3,4-oxadiazole (1f) Brown needles, $\mathrm{m}$. p. $93-95^{\circ} \mathrm{C}$; IR cm ${ }^{-1} 3330,3030,1670,1607(\mathrm{C}=\mathrm{N}-\mathrm{N}=\mathrm{C}), 1267,1068$ (C-O-C), $780 ; \mathrm{H}^{1} \mathrm{NMR}\left(400 \mathrm{MHz}, \mathrm{CDCl}_{3}\right) 7.75\left(\mathrm{~s}, 2 \mathrm{H}, \mathrm{NH}_{2}\right), 7.25-7.69(\mathrm{~m}, \mathrm{~J}=2.6$ and $5.6 \mathrm{~Hz}, 8 \mathrm{H}) ; \mathrm{C}^{13} \mathrm{NMR}\left(75 \mathrm{MHz}, \mathrm{CDCl}_{3}\right) 172.4,149.5,139.2,131.5,128.3$, 125.3; m/z (ES-MS) 295.0, Mol. Formula $\mathrm{C}_{16} \mathrm{H}_{10} \mathrm{~N}_{3} \mathrm{OCl}$, Mol. Wt.: 295.7, Calculated C, 64.95; H, 3.38; N, 14.20; Cl, 12.00, found C, 64.12; H, 3.11; N, $14.05 ; \mathrm{Cl}, 11.66$.

2-amino-5-(5-chloro-2-nitro)phenyl-1,3,4-oxadiazole (1g) Dark yellowish needles, m. p. $71-73{ }^{\circ} \mathrm{C}$; IR $\mathrm{cm}^{-1} 3341,3035,1614(\mathrm{C}=\mathrm{N}-\mathrm{N}=\mathrm{C})$, 1580, 1273, 1071 (C-O-C), 860, 735; $\mathrm{H}^{1} \mathrm{NMR}\left(400 \mathrm{MHz}, \mathrm{CDCl}_{3}\right) 7.75$ (s, $\left.2 \mathrm{H}, \mathrm{NH}_{2}\right), 7.25-7.69(\mathrm{~m}, \mathrm{~J}=2.6$ and $5.6 \mathrm{~Hz}, 3 \mathrm{H}) ; \mathrm{C}^{13} \mathrm{NMR}\left(75 \mathrm{MHz}, \mathrm{CDCl}_{3}\right)$ $172.3,148.3,147.1,146.8,140.8,136.8,130.4,121.2 ; \mathrm{m} / \mathrm{z}$ (ES-MS) 239.7, Mol. Formula $\mathrm{C}_{8} \mathrm{H}_{5} \mathrm{~N}_{4} \mathrm{O}_{3} \mathrm{Cl}$, Mol. Wt.: 240.6, Calculated C, 39.90; H, 2.07; N, $23.27 ; \mathrm{Cl}, 14.75$, found $\mathrm{C}, 39.45 ; \mathrm{H}, 1.95 ; \mathrm{N}, 22.95 ; \mathrm{Cl}, 14.52$.

2-amino-5-(5-chloro-2-hydroxy)phenyl-1,3,4-oxadiazole (1h) Yellow needles, m. p. $78-80{ }^{\circ} \mathrm{C}$; IR $\mathrm{cm}^{-1} 3440,3261,3045,1611(\mathrm{C}=\mathrm{N}-\mathrm{N}=\mathrm{C}), 1276$, 1073 (C-O-C), 1050, 760; H $\mathrm{H}^{1} \mathrm{NMR}\left(400 \mathrm{MHz}, \mathrm{CDCl}_{3}\right) 7.75$ (s, 2H, NH$)_{2}$, 6.46-7.70 (m, J= 2.6 and $5.6 \mathrm{~Hz}, 3 \mathrm{H}), 6.09(\mathrm{~s}, 1 \mathrm{H}, \mathrm{OH}) ; \mathrm{C}^{13} \mathrm{NMR}(75 \mathrm{MHz}$, $\left.\mathrm{CDCl}_{3}\right) 171.9,166.2,151.3,128.7,128.5,128,116.8 ; \mathrm{m} / \mathrm{z}$ (ES-MS) 211.2, Mol. Formula $\mathrm{C}_{10} \mathrm{H}_{8} \mathrm{~N}_{3} \mathrm{OCl}$, Mol. Wt.: 211.6, Calculated C, 56.71; H, 3.78; N, $19.84 ; \mathrm{Cl}, 16.77$, found C, $55.82 ; \mathrm{H}, 3.48 ; \mathrm{N}, 19.65 ; \mathrm{Cl}, 16.55$.

2-amino-5-(4-cyano)phenyl-1,3,4-oxadiazole (4i) Yellow crystals, m. p. 69-71 ${ }^{\circ} \mathrm{C}$; IR cm $\mathrm{cm}^{-1} 3330,3060,2230,1620(\mathrm{C}=\mathrm{N}-\mathrm{N}=\mathrm{C}), 1265,1064$ (C-O-C), 960; $\mathrm{H}^{1} \mathrm{NMR}\left(400 \mathrm{MHz}, \mathrm{CDCl}_{3}\right) 7.75\left(\mathrm{~s}, 2 \mathrm{H}, \mathrm{NH}_{2}\right), 6.54-7.52$ (m, J=2.6 and $5.6 \mathrm{~Hz}, 4 \mathrm{H}) ; \mathrm{C}^{13} \mathrm{NMR}\left(75 \mathrm{MHz}, \mathrm{CDCl}_{3}\right) 171.9,166.1,145.8,132.5,128.6$, $128.5,125,111 ; \mathrm{m} / \mathrm{z}$ (ES-MS) 185.4, Mol. Formula $\mathrm{C}_{0} \mathrm{H}_{6} \mathrm{~N}$, Mol. Wt.: 186.2, Calculated C, 58.06; H, 3.22; N, 30.10, found C, 57.55; H, 3.10; N, 29.76 .

2-amino-5-(3,5-dibromo-2-hydroxy)phenyl-1,3,4-oxadiazole (1j) Black needles, m. p. $88-89{ }^{\circ} \mathrm{C}$; IR cm ${ }^{-1} 3440,3360,3045,1610(\mathrm{C}=\mathrm{N}-\mathrm{N}=\mathrm{C}), 1266$, 1071 (C-O-C), 980, 890, 755, 655; $\mathrm{H}^{1} \mathrm{NMR}\left(400 \mathrm{MHz}, \mathrm{CDCl}_{3}\right) 7.75$ (s, 2H, $\left.\mathrm{NH}_{2}\right), 7.25-7.69(\mathrm{~m}, \mathrm{~J}=2.6$ and $5.6 \mathrm{~Hz}, 2 \mathrm{H}), 6.09(\mathrm{~s}, 1 \mathrm{H}, \mathrm{OH}) ; \mathrm{C}^{13} \mathrm{NMR}(75$ $\mathrm{MHz}, \mathrm{CDCl}_{3}$ ) 158.9, 147.5, 141.6, 136.2, 134.2, 129.9, 124, 123.7; m/z (ESMS) 344.3, Mol. Formula $\mathrm{C}_{10} \mathrm{H}_{7} \mathrm{~N}_{3} \mathrm{OBr}_{2}$, Mol. Wt.: 344.9, Calculated C, 34.78; $\mathrm{H}, 2.02 ; \mathrm{N}, 12.17 ; \mathrm{Br}, 46.37$, found $\mathrm{C}, 34.10 ; \mathrm{H}, 1.88 ; \mathrm{N}, 12.03 ; \mathrm{Br}, 46.11$.

2-amino-5-(3,5-di-tert-butyl-4-hydroxy)phenyl-1,3,4-oxadiazole (1k) Brownish crystals, m. p. $83-85^{\circ} \mathrm{C}$; IR cm ${ }^{-1} 3444,3360,2860,1605(\mathrm{C}=\mathrm{N}-$ $\mathrm{N}=\mathrm{C}), 1273,1063(\mathrm{C}-\mathrm{O}-\mathrm{C}), 1130,749.5 ; \mathrm{H}^{1} \mathrm{NMR}(400 \mathrm{MHz}, \mathrm{CDCl}) 7.75$ (s, $\left.2 \mathrm{H}, \mathrm{NH}_{2}\right), 6.52-7.14(\mathrm{~m}, \mathrm{~J}=2.6$ and $5.6 \mathrm{~Hz}, 3 \mathrm{H}), 1.3\left(\mathrm{~s}, 18 \mathrm{H}, \mathrm{CH}_{3}\right) ; \mathrm{C}^{13}$ $\operatorname{NMR}\left(75 \mathrm{MHz}, \mathrm{CDCl}_{3}\right) 172.4,167,147.5,137.5,132.6,128.5,140.2,138.9$, 42.1, 20.6; m/z (ES-MS) 288.6, Mol. Formula $\mathrm{C}_{16} \mathrm{H}_{23} \mathrm{~N}_{3} \mathrm{O}_{2}$ Mol. Wt.: 289.4, Calculated C, 66.43; H, 7.95; N, 14.53, found C, 65.88; H, 7.56; N, 14.36. 2-amino-5-(2,4-dichloro)phenyl-1,3,4-oxadiazole (11) Pale yellow needles, m. p. 89-91 ${ }^{\circ} \mathrm{C}$; IR cm ${ }^{-1} 3360,3045,1608(\mathrm{C}=\mathrm{N}-\mathrm{N}=\mathrm{C}), 1267,1069$ (C-O-C), 775, 655, 600; $\mathrm{H}^{1} \mathrm{NMR}\left(400 \mathrm{MHz}, \mathrm{CDCl}_{3}\right) 7.75$ (s, 2H, NH ), 6.94$7.04(\mathrm{~m}, \mathrm{~J}=2.6$ and $5.6 \mathrm{~Hz}, 3 \mathrm{H}) ; \mathrm{C}^{13} \mathrm{NMR}(75 \mathrm{MHz}, \mathrm{CDCl}) 164.5,157$, $143,135,133.5,131.7,128.7,128.5 ; \mathrm{m} / \mathrm{z}$ (ES-MS) 229.7, Mol. Formula $\mathrm{C}_{8} \mathrm{H}_{5} \mathrm{~N}_{3} \mathrm{OCl}_{2}$, Mol. Wt.: 230.0, Calculated C, 41.92; H, 2.18; N, 18.34; Cl, 30.56 , found $\mathrm{C}, 41.25 ; \mathrm{H}, 2.03 ; \mathrm{N}, 18.17 ; \mathrm{Cl}, 30.27$.

2-amino-5-(2,3-difluoro)phenyl-1,3,4-oxadiazole (1m) Dark brownish needles, m. p. 80-82 ${ }^{\circ} \mathrm{C}$; IR cm ${ }^{-1} 3360,3050,1611(\mathrm{C}=\mathrm{N}-\mathrm{N}=\mathrm{C}), 1269,1064$ (C-O-C), 935, 765; $\mathrm{H}^{1} \mathrm{NMR}\left(400 \mathrm{MHz}, \mathrm{CDCl}_{3}\right) 7.75\left(\mathrm{~s}, 2 \mathrm{H}, \mathrm{NH}_{2}\right), 6.94-$ $7.04(\mathrm{~m}, \mathrm{~J}=2.6$ and $5.6 \mathrm{~Hz}, 4 \mathrm{H}) ; \mathrm{C}^{13} \mathrm{NMR}\left(75 \mathrm{MHz}, \mathrm{CDCl}_{3}\right) 164.5,162.5$, 142.5, 140.6, 122.2, 111.0, 109.7, 100.1; m/z (ES-MS) 196.6, Mol. Formula $\mathrm{C}_{8} \mathrm{H}_{5} \mathrm{~N}_{3} \mathrm{~F}_{2} \mathrm{O}$, Mol. Wt.: 197.1, Calculated C, 48.73; H, 2.53; N, 21.31; F, 19.28, found $\mathrm{C}, 48.12 ; \mathrm{H}, 2.45 ; \mathrm{N}, 21.18 ; \mathrm{F}, 19.03$.

2-amino-5-(2,6-difluoro)phenyl-1,3,4-oxadiazole (1n) Dark brownish needles, m. p. 82-83 ${ }^{\circ} \mathrm{C}$; IR cm ${ }^{-1} 3360,3055,1601(\mathrm{C}=\mathrm{N}-\mathrm{N}=\mathrm{C}), 1273,1068$ (C-O-C), 950, 790; $\mathrm{H}^{1} \mathrm{NMR}\left(400 \mathrm{MHz}, \mathrm{CDCl}_{3}\right) 7.75$ (s, 2H, NH) 6.94 $7.04(\mathrm{~m}, \mathrm{~J}=2.6$ and $5.6 \mathrm{~Hz}, 4 \mathrm{H}) ; \mathrm{C}^{13} \mathrm{NMR}\left(75 \mathrm{MHz}, \mathrm{CDCl}_{3}\right) 165,162.7$, 142.5, 141.0, 122.2 111.5, 109.7, 100.2; m/z (ES-MS) 196.4, Mol. Formula $\mathrm{C}_{8} \mathrm{H}_{5} \mathrm{~N}_{2} \mathrm{~F}_{2} \mathrm{O}$, Mol. Wt.: 197.1, Calculated C, 48.73; H, 2.53; N, 21.31; F, 19.28, found $\mathrm{C}, 48.20 ; \mathrm{H}, 2.35 ; \mathrm{N}, 21.10 ; \mathrm{F}, 19.05$.

Biological Activity

All the title compounds were tested for their antimicrobial activity by adopting the experimental method of Benson ${ }^{32}$. Whatman No.1 filter paper discs of $6 \mathrm{~mm}$ diameter, placed in a Petri dish, were autoclaved. The test compounds in measured quantities $(1.0 \mathrm{mg}, 0.5 \mathrm{mg})$ were dissolved in $5 \mathrm{~mL}$ of dimethylformamide to produce $200 \mathrm{ppm}$ and $100 \mathrm{ppm}$ solutions, respectively. The filter paper discs were allowed to dry and the amount of the substance per disc was taken as 500 and $250 \mu \mathrm{g}$. The bacterial $(24 \mathrm{~h})$ and fungal $(48 \mathrm{~h})$ cultures from the slants were diluted with sterile water and mixed thoroughly to prepare a clear homogeneous suspension. These suspensions were uniformly spread on solidified agar (nutrient and potato dextrose agar) medium. The filter paper discs prepared from dimethylformamide medium were carefully placed over the spreaded cultures and incubated at $37{ }^{\circ} \mathrm{C}$ for $24 \mathrm{~h}$ for bacteria and at $28-30{ }^{\circ} \mathrm{C}$ for $48 \mathrm{~h}$ for fungi. Paper discs treated with dimethylformamide alone served as control. After the incubation period the plates were examined for inhibition zones. The diameters of inhibition zones (including the diameter of the disc) were measured. All determinations were made in triplicate for each of the compounds and the average value was taken.

\section{RESULTS AND DISCUSSION}

The organic synthesis involving electrochemical techniques under suitable solvents and electrolytes are the basic requirements. The solution phase methods, while successful, were deemed not readily amenable to high throughout synthesis, and thus did not meet our needs. Considering the importance of 1,3,4-oxadiazoles and methods of preparation, 2-amino-5substituted-1,3,4-oxadiazoles were synthesized by electrooxidative cyclization of semicarbazone as an environmentally benign synthetic method in which aforementioned troublesome were removed and minimized. The electroorganic synthesis of 1,3,4-oxadiazole derivatives $\mathbf{1}$ is electrochemical cyclization by electrooxidation of semicarbazone 4. The electrochemical oxidation has various merits. The electrooxidation does not require oxidizing reagents and can be performed at room temperature. Electricity provides chemical processes with special attributes, such as enhanced reaction rate, higher yield of pure products, better selectivity and several ecofriendly advantages.

This electrochemical cyclization gives the oxadiazoles (Scheme1). Acetic acid was used as a solvent and lithium perchlorate as an electrolyte. 


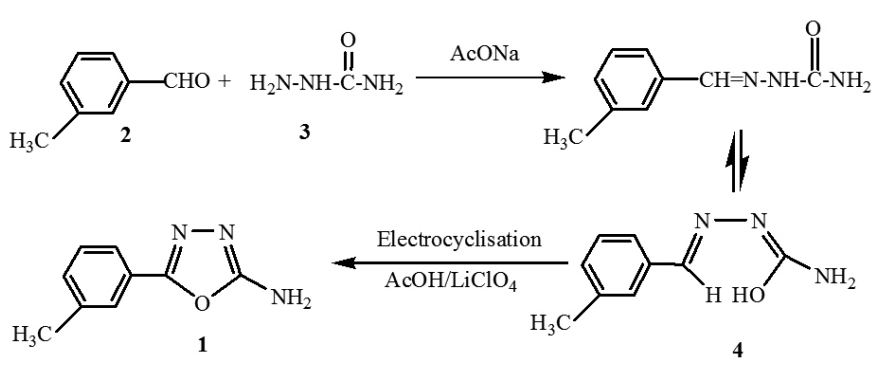

Scheme 1: Synthesis of oxadiazoles 2-amino-5-substituted-1,3,4oxadiazoles. Reagents and conditions: (a) sodium acetate; (b) $\mathrm{CH}_{3} \mathrm{COOH}$, $\mathrm{LiClO}_{4} ; 25^{\circ} \mathrm{C}$.

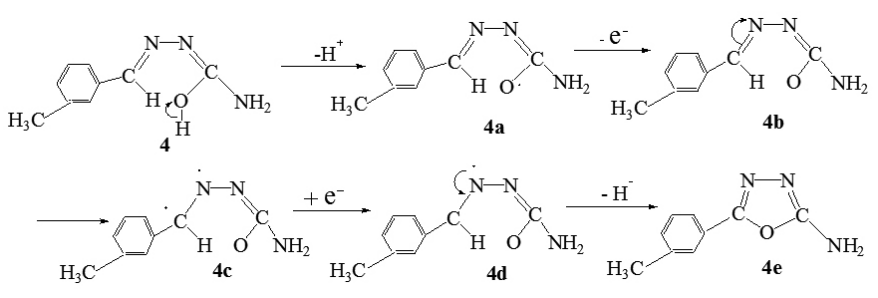

Scheme 2. Mechanistic proposal.

Novel 2-amino-5-substituted-1,3,4-oxadiazoles have been synthesized in excellent yields using the synthetic route outlined in Scheme 1. IR, ${ }^{1} \mathrm{H}$ NMR, ${ }^{13} \mathrm{C}$ NMR and mass spectral data are in agreement with the proposed structures of all synthesized compounds. Lack of ${ }^{1} \mathrm{H}$ NMR resonances observed with $\mathrm{NH}$ and $\mathrm{NH}_{2}$ functions in the ${ }^{1} \mathrm{H}$ NMR spectrum of 1a-n proved that ring closure starting from 4 resulted in the formation of 2-amino-1,3,4-oxadiazole ring. This was further substantiated by the ${ }^{13} \mathrm{C}$ NMR data of $\mathbf{1}$ which showed the peaks at $\delta 170-173$ and $145-150$ due to $\mathrm{C}_{2}$ and $\mathrm{C}_{5}$ of oxadiazole respectively. The IR spectrum shows $1600-1620 \mathrm{~cm}^{-1}$ for $(\mathrm{C}=\mathrm{N}-\mathrm{N}=\mathrm{C})$ and $1063-1073$ $\mathrm{cm}^{-1}$ for $(\mathrm{C}-\mathrm{O}-\mathrm{C})$ in the compounds $1 \mathbf{a}-1 \mathbf{n}$ which confirmed the synthesis of 1,3,4-oxadiazoles.

\section{Antibacterial Activity}

The antibacterial activity of compounds $\mathbf{1 a - 1} \mathbf{n}$ was studied against the growth of Klebsiella pneumoniae, Escherichia coli, (Gram-negative) and Bacillus subtilis, Streptococus aureus (Gram-positive) organisms at the two concentrations (25 and $50 \mathrm{ppm}$ ) taking Streptomycin as the standard (Table 2). The majority of the compounds exhibited significant (good) antibacterial activity against $E$. coli, K. pneumonia, B. subtilis and S. aureus as compared to Streptomycin. The screening results of antibacterial activity revealed that compounds $\mathbf{1 c}$ and $\mathbf{1 j}$ exhibited approximately similar activity to the standard Streptomycin. Compounds $\mathbf{1 f}, \mathbf{1 g}, \mathbf{1 h}, \mathbf{1 i}$ and $\mathbf{1 l}$ exhibited slightly less antibacterial activity. Remaining compounds exhibited weak antibacterial activity against all bacterial strains used for our evaluation.

Antifungal Activity

The compounds $\mathbf{1 a}-\mathbf{1} \mathbf{n}$ were screened for their antifungal activity against Aspergillus niger and C. pannical species along with the standard fungicide Griseofulvins 1a-1n (Table 3). The disc diffusion method was followed for screening the compounds at three concentrations (10,100 and $1000 \mathrm{ppm})$. The screening results revealed that all the compounds displayed good antifungal activity against $A$. niger and $C$. pannical. However, compounds $\mathbf{1 c}$ and $\mathbf{1} \mathbf{j}$ showed equal antifungal activity when compared with the Griseofulvins.

The antimicrobial activity of the compounds varied upon the type and position of the substituents at 5-substituted-2-amino-1,3,4-oxadiazole moiety. It can be concluded from the antimicrobial screening results that when 5-substituted-2-amino-1,3,4-oxadiazoles were substituted with aryl halide the antimicrobial activity was altered to an appreciable extent.
Table 2. Antibacterial activity of compounds 1a-1n

\begin{tabular}{|ccccccccc|}
\hline \multirow{2}{*}{ Compound } & \multicolumn{7}{c|}{ Zone of inhibition (mm) } \\
\cline { 2 - 9 } & \multicolumn{2}{c}{ E. coli } & $\begin{array}{c}\text { K. } \\
\text { pneumonia }\end{array}$ & B. subtilis & S. aureus \\
\cline { 2 - 9 } & $\begin{array}{c}25 \\
\text { ppm }\end{array}$ & $\begin{array}{c}50 \\
\text { ppm }\end{array}$ & $\begin{array}{c}25 \\
\text { ppm }\end{array}$ & $\begin{array}{c}50 \\
\text { ppm }\end{array}$ & $\begin{array}{c}25 \\
\text { ppm }\end{array}$ & $\begin{array}{c}50 \\
\text { ppm }\end{array}$ & $\begin{array}{c}25 \\
\text { ppm }\end{array}$ & $\begin{array}{c}50 \\
\text { ppm }\end{array}$ \\
\hline 1a & 8 & 14 & 11 & 17 & 10 & 15 & 12 & 15 \\
$\mathbf{1 b}$ & 10 & 15 & 10 & 16 & 12 & 18 & 13 & 17 \\
$\mathbf{1 c}$ & 17 & 22 & 18 & 21 & 18 & 23 & 16 & 22 \\
$\mathbf{1 d}$ & 12 & 17 & 9 & 13 & 12 & 15 & 11 & 15 \\
$\mathbf{1 e}$ & 10 & 15 & 9 & 14 & 12 & 16 & 13 & 16 \\
$\mathbf{1 f}$ & 16 & 20 & 17 & 21 & 15 & 20 & 14 & 19 \\
$\mathbf{1 g}$ & 17 & 21 & 14 & 20 & 16 & 20 & 16 & 20 \\
$\mathbf{1 h}$ & 14 & 19 & 16 & 19 & 15 & 18 & 17 & 20 \\
$\mathbf{1 i}$ & 15 & 18 & 14 & 18 & 13 & 17 & 17 & 19 \\
$\mathbf{1 j}$ & 18 & 22 & 18 & 23 & 17 & 22 & 18 & 23 \\
$\mathbf{1 k}$ & 12 & 15 & 12 & 16 & 10 & 14 & 13 & 17 \\
$\mathbf{1 1}$ & 17 & 20 & 16 & 20 & 18 & 21 & 15 & 19 \\
$\mathbf{1 m}$ & 14 & 18 & 13 & 17 & 14 & 18 & 12 & 16 \\
$\mathbf{1 n}$ & 15 & 18 & 12 & 16 & 13 & 18 & 13 & 17 \\
Streptomycin & 20 & 23 & 19 & 24 & 19 & 24 & 19 & 23 \\
Control & & & & & & & & \\
(DMF) & 0 & 0 & 0 & 0 & 0 & 0 & 0 & 0 \\
\hline & & & & & & & & \\
\hline
\end{tabular}

Table 3. Antifungal activity of the compounds $\mathbf{1 a - 1 n *}$

\begin{tabular}{|c|c|c|c|c|c|c|}
\hline \multirow{2}{*}{ Compound } & \multicolumn{3}{|c|}{ A. niger } & \multicolumn{3}{|c|}{ C. pannical } \\
\hline & $\begin{array}{c}10 \\
\mathrm{ppm}\end{array}$ & $\begin{array}{l}100 \\
\text { ppm }\end{array}$ & $\begin{array}{l}1000 \\
\mathrm{ppm}\end{array}$ & $\begin{array}{c}10 \\
\mathrm{ppm}\end{array}$ & $\begin{array}{r}100 \\
\mathrm{ppm}\end{array}$ & $\begin{array}{l}1000 \\
\mathrm{ppm}\end{array}$ \\
\hline $1 \mathrm{a}$ & 19 & 42 & 73 & 19 & 41 & 74 \\
\hline $1 b$ & 18 & 42 & 72 & 21 & 45 & 77 \\
\hline $1 \mathrm{c}$ & 44 & 58 & 98 & 45 & 57 & 98 \\
\hline 1d & 20 & 44 & 72 & 22 & 43 & 75 \\
\hline $1 e$ & 17 & 36 & 63 & 18 & 37 & 65 \\
\hline 1f & 38 & 56 & 97 & 40 & 51 & 96 \\
\hline $1 \mathrm{~g}$ & 40 & 53 & 96 & 42 & 53 & 97 \\
\hline $1 \mathrm{~h}$ & 41 & 51 & 96 & 39 & 54 & 96 \\
\hline $1 \mathbf{i}$ & 21 & 44 & 70 & 20 & 43 & 68 \\
\hline $1 \mathrm{j}$ & 46 & 59 & 98 & 46 & 58 & 98 \\
\hline $1 \mathrm{k}$ & 26 & 45 & 71 & 23 & 46 & 75 \\
\hline 11 & 42 & 55 & 95 & 41 & 55 & 97 \\
\hline $1 \mathrm{~m}$ & 33 & 45 & 88 & 21 & 39 & 84 \\
\hline 1n & 32 & 46 & 87 & 22 & 42 & 85 \\
\hline Griseofulvin & 66 & 86 & 100 & 65 & 83 & 100 \\
\hline $\begin{array}{l}\text { Control } \\
\text { (DMF) }\end{array}$ & 0 & 0 & 0 & 0 & 0 & 0 \\
\hline
\end{tabular}
liter $\left.^{-1}\right)$

*Average inhibition of fungal growth (\%) at stated concentration (mg/ 


\section{CONCLUSION}

In conclusion we have developed a convenient method for the synthesis of novel 2-amino-5-substituted-1,3,4-oxadiazole derivatives in excellent yields which is not so easy to achieve by chemical methods. These compounds exhibited significant activity against the growth of bacterial and fungal strain. The antimicrobial activity of the compounds varied upon the type and position of the substituents at 5-substituted-2-amino-1,3,4-oxadiazole moiety. It is evident from the antimicrobial screening results that when 5-substituted-2amino-1,3,4-oxadiazoles were substituted with aryl halide the antimicrobial activity was altered to an appreciable extent.

\section{ACKNOWLEDGEMENTS}

The author thanks to Sophisticated Analytical Instrument Facility (SAIF) a division of CDRI (Central Drug Research Institute) Lucknow for recording spectra and microanalyses.

\section{REFERENCES}

1. M. Tyagi, A. Kumar, Oriental. J. Chem., 18, 125, (2002).

2. B. S. Holla, R. Gonaslaves, S. Shenoy, Eur. J. Med. Chem., 35, 267, (2000).

3. N. Cesur, S. Birteksoz, G. Otuk, Acta. Pharm. Turcica, 44, 23, (2002).

4. U. V. Laddi, S. R. Desai, R. S. Bennur, S. C. Bennur, Indian J. Heterocycl. Chem., 11, 319, (2002).

5. X. Zou, Z. Zhang, G. Jin, J. Chem. Res. Synopses., 228 (2002).

6. X. J. Zou, L. H. Lai, G. Y. Jin, Z. X. Zhang, J. Agric. Food Chem., 50, 3757, (2002).

7. E. Palaska, G. Sohin, P. Kclicen, N. T. Darlu, G. Altinok, Farmaco, 57, 101 (2002).

8. A. Afiatpour, R. M. Srivastava, M. L. Oliveira, E. J. Barreiro, Braz. J. Med. Biol. Res., 27, 1403, (1994).

9. L. B. Clapp, A. R. Katritzky, C. W. Rees Eds., Comprehensive Heterocyclic Chemistry, Pergamon Press, Oxford, 1984.

10. R. S. Gani, S. S. Pujar, G. S. Gadaginamath, Indian J. Heterocycl. Chem., 12, 25, (2002).
11. S. M. Golovlyova, Y. A. Moskvichey, E. M. Alov, D. B. Kobylinsley, V. V. Ermolaeva, Chem. Heterocycl. Compd., 37, 1102, (2001).

12. F. M. Liu, B. L. Wang, Z. F. Zhang, Youji Huaxue, 21, 1126, (2001).

13. O. M. Aboulwafa, A. M. Omar, Sulfur Lett., 14, 181, (1992).

14. H. M. Faidallah, E. M. Sharshira, S. A. Basaif, A. E. A-Ba-Oum, Phos. Sulf. Sil. Rel. Elem., 67, 177, (2002).

15. A. Hetzheim, K. Moeckel, Adv. Heterocyclic Chem., 07, 183, (1966).

16. J. Hill, In: K.T. Potts Eds., Comprehensive Heterocyclic Chemistry, Pergamon Press, Oxford, 06, 427, (1984).

17. F. T. Cappo, K. A. Evans, T. L. Graybill, G. Burton, Tetrahedron Lett., 45, 3257, (2004)

18. A. Kumar, S. Kumar, R. K. P. Singh, Proc. Nat. Acad. Sci. India, 75A IV, 233, (2005)

19. K. L. Yadav, A. Kumar, S. Kumar, R. K. P. Singh, Transactions of the SAEST, 40, 106, (2005).

20. S. Kumar, R. K. P. Singh, J. Indian Chem. Soc., 82, 934, (2005).

21. S. Kumar, P. Yadav, R. K. P. Singh, Transactions of the SAEST, 41, 05, (2006).

22. K. L. Yadav, S. Kumar, A. Kumar, R. K. P. Singh, J. Indian Chem. Soc., 81, 595, (2004)

23. L. K. Sharma, S. Kumar, P. Yadav, R. K. P. Singh, Indian J Chem., Sec. B, 47, 1277, (2008).

24. C. K. Mann, K. K. Barnes, Electrochemical Reactions In Non aqueous Systems, Marcel Dekker, Inc. New York, 1970.

25. A. J. Fry, Synthetic Organic Electrochemistry, Wiley-Interscience Publication, New York, 1989.

26. T. Shono, Electroorganic Synthesis, Academic Press Ltd., London, 1991.

27. S. Kumar, L. K. Sharma, R. K. P. Singh, J. Indian Chem. Soc., 83, 1160 (2006).

28. L. K. Sharma, S. Kumar, R. K. P. Singh, Transactions of the SAEST, 41 48, (2006)

29. K. L. Yadav, A. Kumar, S. Kumar, R. K. P. Singh, J Electrochem. Soc., 52(3), 114, (2003)

30. S. Kumar, J. Korean Chem. Soc., 53, 159, (2009).

31. S. Singh, S. Kumar, L. K. Sharma, R. K. P. Singh, J. Indian Chem. Soc., 86, 734, (2009).

32. H. J. Benson, Microbiological Applications, 5 th Eds., W. C. Brown Publications, Boston, MA, USA, (1990). 